\title{
Photosensitivity reaction induced by erlotinib
}

\author{
Yuhei Tokimasa $\cdot$ Keiichi Fujiwara $\cdot$ Hisao Higo $\cdot$ Nobuhisa Kameyama \\ Hiroe Kayatani - Ken Sato • Kiyoshi Matsuo • Toshiro Yonei • \\ Keiko Manabe $\cdot$ Yoko Yasui $\cdot$ Kenji Asagoe $\cdot$ Toshio Sato
}

Received: 6 February 2012/ Accepted: 16 March 2012/Published online: 2 May 2012

(C) The Japan Society of Clinical Oncology 2012

\begin{abstract}
We describe the case of a 70-year-old man with non-small-cell lung cancer who received erlotinib and developed skin eruptions. He noted erythema on his face, head, neck, a V-shaped area of his chest, and the dorsum of his hands after exposure to sunlight 12 weeks after the beginning of erlotinib treatment. The skin eruption spread only on sunexposed areas. Histological findings showed lichenoid dermatitis with lymphocyte infiltration, and a reduced minimal erythema dose was observed. Photo patch testing revealed a positive reaction, confirming erythema to be a photosensitivity reaction to erlotinib. The photosensitivity reaction is a rare toxicity in erlotinib treatment. It is important to distinguish a photosensitivity reaction from a common skin rash due to erlotinib such as acneiform eruptions.
\end{abstract}

Keywords Photosensitivity reaction - Erythema . Erlotinib - Minimal erythema dose $\cdot$ Photo patch test

\section{Introduction}

Erlotinib is an epidermal growth factor receptor tyrosine kinase (EGFR-TK) inhibitor that has an antitumor activity for non-small-cell lung cancer (NSCLC). Treatment with

Y. Tokimasa $\cdot$ K. Fujiwara $(\bowtie) \cdot$ H. Higo $\cdot$ N. Kameyama

H. Kayatani - K. Sato - K. Matsuo - T. Yonei - T. Sato

Department of Respiratory Medicine, National Hospital

Organization Okayama Medical Center, 1711-1 Tamasu,

Kita-ku, Okayama 701-1192, Japan

e-mail: keiichi@okayama3.hosp.go.jp

K. Manabe $\cdot$ Y. Yasui $\cdot$ K. Asagoe

Department of Dermatology, National Hospital Organization

Okayama Medical Center, 1711-1 Tamasu,

Kita-ku, Okayama 701-1192, Japan
EGFR-TK inhibitors is associated with a decreased incidence of systemic adverse effects in comparison with standard chemotherapeutic drugs [1]. In contrast, dermatologic toxic effects by EGFR-TK inhibitors are the most common events, including acneiform skin rashes, hyperpigmentation, xerotic skin, pruritus, skin fissures, nail changes, and disorders of mucous membranes with an incidence ranging from 49 to $100 \%$ of the patients [2]. Additionally, erlotinib has a unique toxicity of cosmetic hair alteration [3]. However, the photosensitivity reaction has been rarely reported in erlotinib treatment. We present a patient with NSCLC showing a photosensitivity reaction induced by erlotinib.

\section{Case report}

A 70-year-old man receiving erlotinib visited our hospital because of erythema on his face and the dorsum of his hands in June 2011. He had been diagnosed with adenocarcinoma of the lung (T2N2M1, stage IV) in May 2008. At that time he was treated with six cycles of carboplatin and docetaxel, a subsequent four cycles of gemcitabine and vinorelbine, and two cycles of carboplatin, pemetrexed, and bevacizumab. Although his tumor harbored no EGFR mutations, he had been administered $150 \mathrm{mg}$ of erlotinib as fourth line chemotherapy since February 2011. An acneiform skin rash on his face and precordia was shown on day 16 of erlotinib treatment, which was treated with topical steroids, systemic antibiotics, and moisturizer. Thirty-seven days after beginning treatment, erlotinib was interrupted because of grade 3 appetite loss and a severe skin rash that spread over almost the whole body. A skin biopsy revealed the rash to be a pustular drug eruption caused by erlotinib, and the eruption was treated with steroid ointment. The 
treatment with a reduced dose of erlotinib (100 mg daily) was restarted after the eruptions had resolved.

On day 104 when ultraviolet radiation was the strongest in the seasons, diffuse infiltrated erythema was observed on the patient's face, head, neck, a V-shaped area of the chest, and the dorsum of the hand after the exposure to sunlight (Fig. 1). Histological examination of skin biopsy samples showed lichenoid changes such as liquefaction degeneration at the basement membrane zone and dense lymphoid infiltration in the dermis (Fig. 2). Photo testing using 6 or $12 \mathrm{~J} / \mathrm{cm}^{2}$ of ultraviolet A (UVA) caused erythema during the administration of erlotinib, whereas it did not cause erythema 14 days after withdrawal of erlotinib, indicating that the minimal erythema dose (MED) was reduced with the use of erlotinib. Additionally, the photo patch test using UVA showed development of erythema at the site where 10 or $20 \%$ concentration of erlotinib reagent had been applied (Fig. 3). In contrast, the erlotinib patch test without UVA irradiation showed no specific reaction. Consequently, we diagnosed the erythema as a photosensitivity reaction induced by erlotinib. Erlotinib was restarted and continued at $100 \mathrm{mg}$ with the use of sunscreen and the avoidance of sun exposure when possible. Since then, the erythema did not appear again until erlotinib was stopped because of the progression of the lung cancer (Fig. 4).

\section{Discussion}

We experienced a patient with advanced NSCLC showing a photosensitivity reaction due to erlotinib. Initially, the eruptions were recognized as the skin toxicity commonly

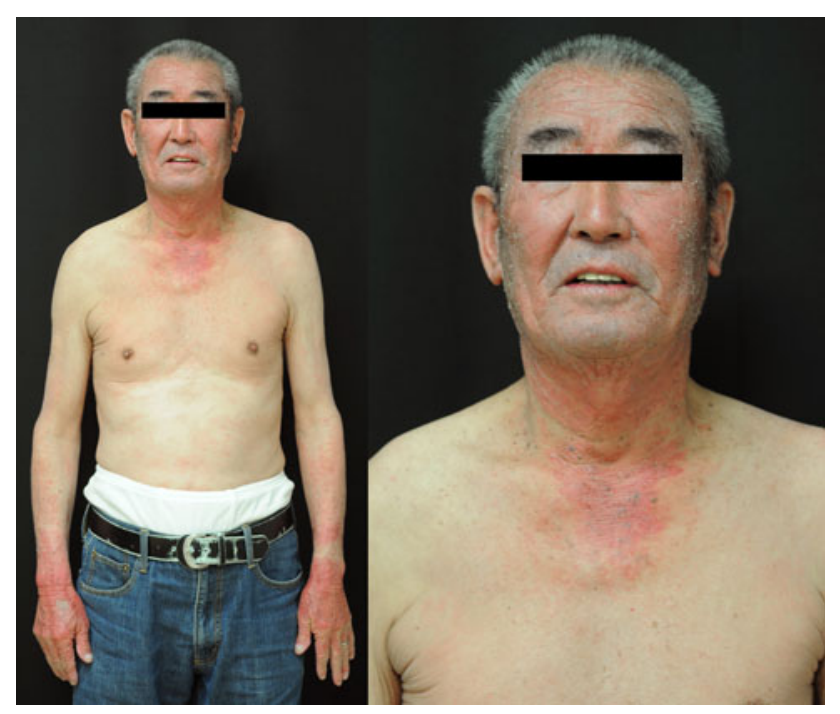

Fig. 1 The erythema spread on his face, head, neck, a V-shaped area of his chest, and dorsum of the hand, which were all photo-exposed areas observed during erlotinib treatment. The event was finally confirmed as a photosensitivity reaction induced by erlotinib according to the distribution of eruptions, pathological findings, reduced MED, and positive reaction of the photo patch test. In published series, there has been only one case
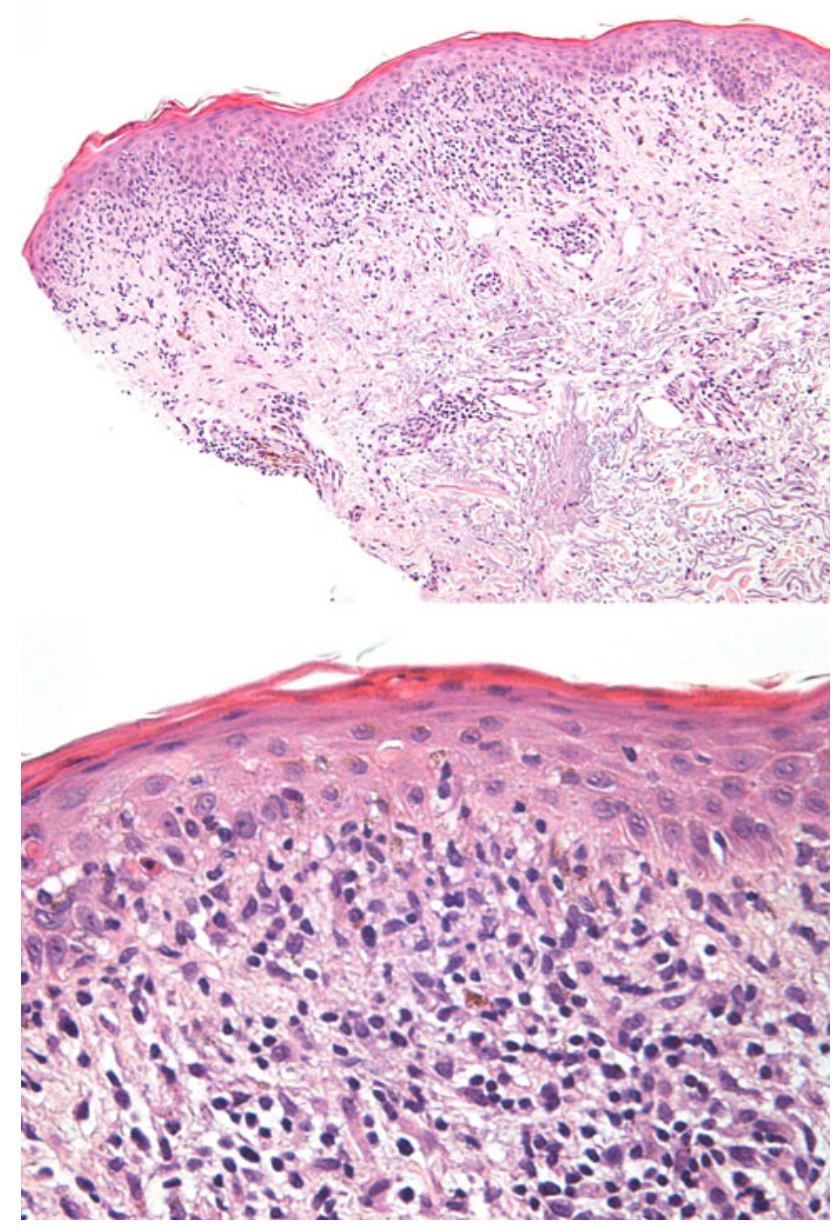

Fig. 2 Histological examination showed liquefaction degeneration at the basement membrane zone and dense lymphoid infiltration in the dermis
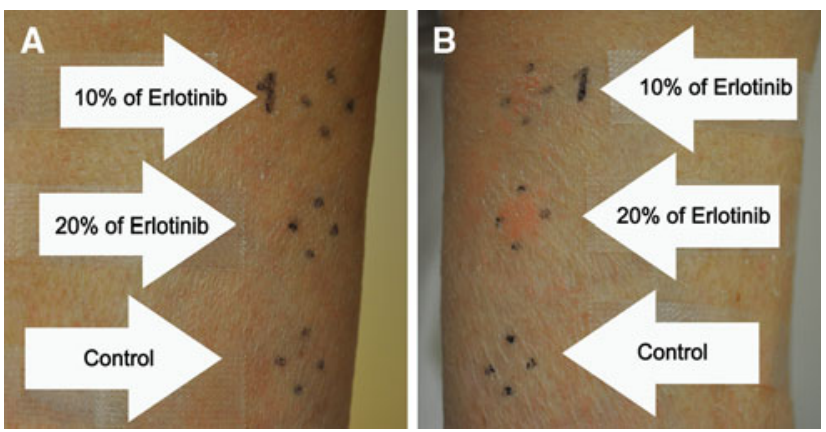

Fig. 3 Patch test showed no specific reaction to erlotinib (a). In contrast, the photo patch test was positive for erlotinib (b) 


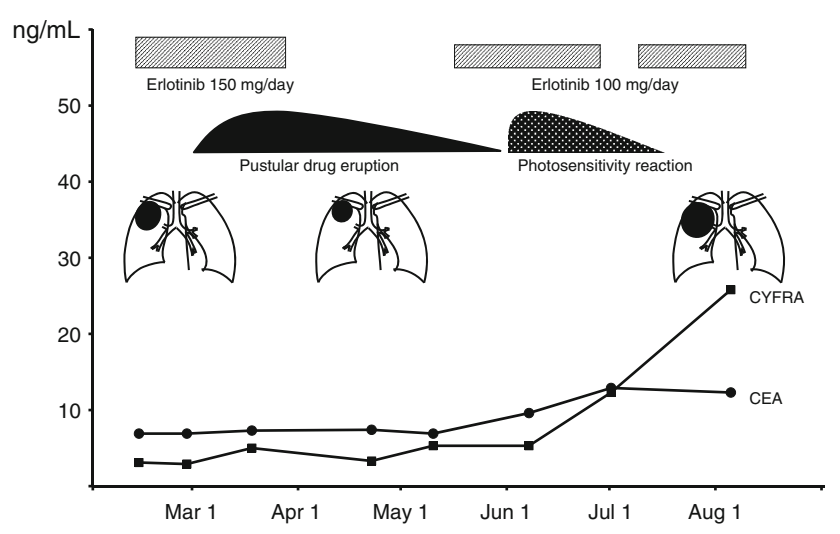

Fig. 4 Clinical course showing the dose and duration of erlotinib, its efficacy, and conditions of skin rash

report of a photosensitive reaction due to an EGFR-TK inhibitor, and the event seems to be an unusual toxicity. Luu et al. [4] described a case of an erlotinib-induced photosensitive rash spreading outside sunscreen-covered areas after sun exposure. In this report, photosensitivity was confirmed by the distribution of eruptions and pathological examinations, but not by photo patch testing. Our case presented characteristic findings of photosensitivity reaction revealed by photo testing.

It is unclear why erlotinib causes a photosensitivity reaction. However, a possible explanation was suggested in previous reports. Recently, several cases of photosensitivity reactions caused by vandetanib, which is an inhibitor of EGFR, in addition to vascular endothelial growth factor receptor and the RET (rearranged during transfection) kinases, have been reported [5-8]. Signaling via the EGFR is critical in keratinocyte responses to ultraviolet radiation (UVR), including proliferation and evasion of apoptosis [9]. UVR combined with EGFR inhibition may cause an additive oxidative stress on keratinocytes and may cause an inflammatory response in the skin, both of which may contribute to the pathogenesis of the skin rash [4]. Accordingly, erlotinib targeting EGFR-TK may also cause a photosensitivity reaction like vandetanib.

There might be quite a few cases showing photosensitivity reactions that we diagnosed as erlotinib-induced common skin rashes. Although a skin rash is not usually a fatal toxicity, it often causes psychosocial discomfort in NSCLC patients, and sometimes needs the interruption or dose reduction of erlotinib. When the physician recognizes a skin eruption during erlotinib treatment and its distribution is in sun-exposed areas, a photosensitive reaction should be considered and be examined in close cooperation with a dermatologist. Once the diagnosis of a photosensitive reaction can be made, avoiding sun exposure will not interrupt the therapy. Additionally, the utilization of sunscreen may prevent photosensitive reactions, although a previous clinical trial of sunscreen for an EGFR-TK inhibitor-induced skin rash failed to demonstrate its usefulness [10]. It seems to be important to educate patients about using adequate sun protection.

Conclusively, as some patients among those receiving erlotinib will show photosensitivity reactions, we need to differentiate photosensitivity reactions from common erlotinib-induced skin rashes.

Conflict of interest The authors declare no conflict of interest, and the study did not receive any funding.

\section{References}

1. Dancey J, Sausville EA (2003) Issues and progress with protein kinase inhibitors for cancer treatment. Nat Rev Drug Discov 2:296-313

2. Potthoff K, Hofheinz R, Hassel JC et al (2011) Interdisciplinary management of EGFR-inhibitor-induced skin reactions: a German expert opinion. Ann Oncol 22:524-535

3. Kudo K, Fujiwara K, Tsushima M et al (2011) Toxicity manifesting as cosmetic hair alterations during erlotinib treatment. Acta Oncol 50:146-148

4. Luu M, Lai SE, Patel J et al (2007) Photosensitive rash due to the epidermal growth factor receptor inhibitor erlotinib. Photodermatol Photoimmunol Photomed 23:42-45

5. Fava P, Quaglino P, Fierro MT et al (2010) Therapeutic hotline. A rare vandetanib-induced photo-allergic drug eruption. Dermatol Ther 23:553-555

6. Chang CH, Chang JW, Hui CY et al (2009) Severe photosensitivity reaction to vandetanib. J Clin Oncol 27:e114-e115

7. Kong HH, Fine HA, Stern JB et al (2009) Cutaneous pigmentation after photosensitivity induced by vandetanib therapy. Arch Dermatol 145:923-925

8. Kiura K, Nakagawa K, Shinkai T et al (2008) A randomized, double-blind, phase IIa dose-finding study of Vandetanib (ZD6474) in Japanese patients with non-small cell lung cancer. J Thorac Oncol 3:386-393

9. Peus D, Vasa RA, Meves A et al (2000) UVB-induced epidermal growth factor receptor phosphorylation is critical for downstream signaling and keratinocyte survival. Photochem Photobiol 72: $135-140$

10. Jatoi A, Thrower A, Sloan JA et al (2010) Does sunscreen prevent epidermal growth factor receptor (EGFR) inhibitor-induced rash? Results of a placebo-controlled trial from the North Central Cancer Treatment Group (N05C4). Oncologist 15:1016-1022 